\section{Clinico-anatomical Analysis of the Oculomotor Fasciculus}

Key words: concomitant skew deviation, magnetic resonance imaging (MRI), ocular tilt reaction, oculomotor fasciculus (oculomotor fascicles), ophthalmoplegia, partial oculomotor nerve palsy

\section{(Intern Med 50: 2075, 2011)}

(DOI: 10.2169/internalmedicine.50.5434)

To the Editor In a recent issue of Internal Medicine, I read with interesting 'Vertical Diplopia due to Midbrain Infarction' reported by Saji and coworkers (1).

It is well known that the human evidence of oculomotor fascicular arrangement in the midbrain is a topic of debate (2-4). In the English language literature, various diagrams showing the intrafascicular anatomy of the oculomotor nerve have been proposed (2-4). These previous reports suggested that the fibers to the inferior oblique muscle and levator palpebrae superioris might run separately in the oculomotor fasciculus (2-4). Here, Saji et al (1) noted that, based on Park's three-step test and Hess chart findings, the inferior oblique muscle and levator palpebrae superioris were involved simultaneously in the left eye due to an inferior midbrain tegmentum infarction on the left side. Whether the sphincter muscle was impaired or not was not discussed in their article (1). Consequently, the authors (1) proposed that the fibers controlling the inferior oblique muscle and levator palpebrae superioris might be located adjacent to each other in the oculomotor fasciculus.
In this patient, Hess chart findings demonstrated concomitant skew deviation with lower position of the left eye (1). Therefore, it is very difficult to conclude whether slight palsies of the superior rectus muscle and the inferior oblique muscle in the left eye exist or not. On the other hand, the actions of the inferior oblique muscle are lateral rotation, elevation and abduction of the eye. However, in this patient, despite the presence of inferior oblique muscle palsy on the left, Hess chart clearly demonstrated restriction of adduction in the left eye (1). Thereby, I believe that palsy of the left medial rectus muscle also occurred in this patient. Based on these facts, I do not agree with Saji and coworkers' hypothesis (1) that the fibers controlling the inferior oblique muscle and levator palpebrae superioris might be located adjacent to each other in the oculomotor fasciculus.

The authors state that they have no Conflict of Interest (COI).

Hiromasa Tsuda

\section{References}

1. Saji N, Tanigawa M, Kita Y, Yokono K. Vertical diplopia due to midbrain infarction. Intern Med 50: 531-532, 2011.

2. Castro O, Johnson LN, Mamourian AC. Isolated inferior oblique paresis from brain-stem infarction. Perceptive on oculomotor fascicular organization in the ventral midbrain tegmentum. Arch Neurol 47: 235-237, 1990.

3. Ksiazek SM, Slamovits TL, Rosen CE, Burde RM, Parisi F. Fascicular arrangement in partial oculomotor paresis. Am J Ophthalmol 118: 97-103, 1994.

4. Schwartz TH, Lycette CA, Yoon SS, Kargman DE. Clinicoradiographic evidence for oculomotor fascicular anatomy. J Neurol Neurosurg Psychiatry 59: 338, 1995. 